\title{
Comparison between Salivary Cortisol Release in Rest and Training Days in Male Fitness Trainee
}

\author{
Ardalan Shariat (corresponding author) \\ Department of Physical Education and Sport Sciences,faculty of educational studies, UPM University \\ Serdang, Selangor, Malaysia \\ Tel:006-0173365494 E-mail: Ardalan_sh2002@yahoo.com \\ Mehdi Kargarfard \\ Department of Exercise Physiology, Faculty of Physical Education and Sport Sciences, Esfahan university \\ Esfahan, Iran \\ Pooya Nekooei \\ Department of Physical Education and Sport Sciences,faculty of educational studies,UPM University \\ Serdang, Selangor, Malaysia \\ Sara Majlesi \\ Department of Physical Education and Sport Sciences,faculty of educational studies,UPM University \\ Serdang, Selangor, Malaysia
}

Received: 01-01- 2013

Accepted: 01-02- 2013

Published: 30-04- 2013

doi:10.7575/aiac.ijkss.v.1n.1p.9

URL: http://dx.doi.org/10.7575/aiac.ijkss.v.1n.1p.9

\begin{abstract}
Background: The aim behind the current study was a comparison between salivary cortisol release in rest and training days in male fitness trainers. Methods: In such balanced crossover research, 15 healthy fitness-trained participants (with the mean age, weight and Height of $20.35 \pm 1.20$ years, $74.15 \pm 3.20 \mathrm{~kg}$ and $177.30 \pm 3.45 \mathrm{~cm}$, respectively) who had a four-year-previous experience in fitness exercise were chosen. While performing the research, the subjects were involved in a fitness training. The protocol contained three times of fitness training in a week, which would totally be alternative days of "rest" and "exercise" in the period of training. It means that they did training on Monday, Wednesday and Friday, and rested on the other days. To ensure the validity of this study, the participants were selected randomly, in both control and experimental groups. In the meantime, the protocol for fitness exercise was made up of four sets of 15 repetitions utilizing $65 \%$ of 1 repetition maximum $(1 \mathrm{RM})$. Within each testing time, saliva samples were taken once in two hours for a maximum of 16 hours. In this study, the ANOVA was followed by a Post-hoc LSD test. Also, the significance level was deemed as $(\mathrm{P} \leqslant 0.05)$. Results: It was shown that there was a statistically significant addition in the salivary cortisol level right away after the fitness exercise session $(\mathrm{P} \leqslant 0.05)$. However, no statistically significant difference was observed between exercise and control days in the circadian rhythm of salivary cortisol $(\mathrm{P}>0.05)$. Conclusion: The present research demonstrated that while there was a significant influence of fitness exercise on cortisol right away after exercise, no statistically significant effect has been observed on the circadian secretion of cortisol in fitness sportsmen.
\end{abstract}

Keywords: fitness exercise; circadian rhythm; salivary cortisol

\section{Introduction}

Based on the available documents and evidences, many of the physiological and psychological variants and their responses to physical exercises are influenced by the changes in the time of the exercise during the day, and the change in the circadian rhythm (Mcguigan, Egan, \& Foster, 2004). Nonetheless, the effects of the circadian rhythm of the endocrine hormones and the responses of the physical immune mechanism to the exercises have not been determined yet. There are still unanswered questions in the studies on the levels of the cortisol and salivary testosterone in response to physical exercises. However, these ambiguities seem to be the results of the sampling time, the type, intensity and duration of the exercise, the fitness level of the subjects, the quality of the food intake and even the circadian rhythm (Kraemer, Acevedo, Dzewaltowski, Kilgore, Kraemer, 1996). One of the laboratory methods used for the analysis of the physiological and psychological condition of the athletes, is the study of the changes in the hormones with anabolic and catabolic characteristics. Therefore, the role of testosterone and cortisol as, respectively, the most important anabolic and catabolic hormones, is considered highly significant. According to the scholars, these hormones are responsive to the physical exercises, physical or mental pressure and stress. If there are not enough rest periods between the exercises, 
the changes in these hormones lead the body to catabolic state. Testosterone is an anabolic hormone which promotes protein synthesis and has an important role in the growth and maintenance of the muscles and tissues. Cortisol is a catabolic and steroid hormone that is primarily involved in the metabolism and physical responses to stress and pressure during exercises. However, prolonged increase of cortisol can disturb the immune system and destroy the proteins (Gettler, McDade, \& Kuzawa, 2011).

So far majority of the studies has used blood samples to identify hormone levels; as Bosco and his collaborator also announced, hormonal plasma samples had so far been used to analyze and determine the impact of exercise in the different sports categories (Bosco, Colli, Bonomi, Duvillard, 2000). In this respect, Fry and his colleagues (Fry et al., 2000), as well as Gotshalk and his colleagues (Daly, Rich, 1998) have also demonstrated the increase in the level of cortisol hormone of the serum after a resistance exercise session, in their academic reports. Cooke and his colleagues have shown in their studies that the increase in the level of cortisol is followed by a decrease in the level of testosterone in the serum (Sciences, 2011).

Because of the advantages of the analysis of blood cortisol over salivary cortisol, and the high consistency $(r>0.9)$ (Rosmond et al., 2003) found between the levels of cortisol and testosterone in plasma and saliva, as well as the fact that no study has so far analyzed the acute effects of fitness exercise protocols on the circadian rhythm of salivary cortisol in men, the evaluation and measurement of the level of these hormones, especially salivary cortisol, can serve as a well-selected method in both basic researchers and clinical environments. It can also be a good measure in programming the sessions of fitness exercise. Therefore, the aim of this study is to make a comparison between salivary cortisol release in rest and training days in male fitness trainers.

\section{Methods}

\subsection{Participants}

A balanced cross sectional study was performed to evaluate the effects of fitness exercise on circadian rhythm of salivary cortisol in waking state. The statistical population of the present study included all the fitness athletes (1230 subjects) who were members of the 8 sport clubs in Esfahan, a city in center of Iran in march of 2010, with at least four years of experience in fitness exercise, three regular sessions of exercise per week and they were the persons who has never used any supplements same proteins, creatin, amino acids and other products that have influence on the result of the study. Only fifteen of the subjects with the aforementioned criteria were purposefully selected as the sample. The numbers of selected subjects were low because most of the clients used supplements similar to creatine, antioxidant, amphitamines, and stimulators to improve their performance, and it can has some effects on our data. Therefore we omitted them . Before starting the protocol, we explained the target and the method of the research tests to the subjects and cleared it for them. Subsequently, we obtained ethics approval from the committee of the Department of Sport Science located in Esfahan University in Iran. Then, they were requested for their high cooperation and attempt for the best performance of the test. They were also asked to follow the natural sleep pattern ( 8 hours a day), normal daily routine/activity and nourishment of the research procedure. Subjects were asked to keep their normal diet and avoid taking any extra food or drink any stimulator beverage containing caffeine or other stimulator that might have any effect on the performance and the results of the research.

\subsection{Measurements}

The height, weight and fat percentage of the athletes were measured, and their Body Mass Index (BMI) was determined. In order to ensure the accuracy of the results of these measurements, the weight and height of the subjects were measured at three intervals during the day and then the average was recorded as the result. For measuring the height, Secasatadiometer with an accuracy of $0.5 \mathrm{~cm}$, and the weight, Seca scale were used. For the measurement of the fat percentage of the three fatty areas of skin folds of abdomen, Triceps and Upper iliaic, the Lunge Caliper with an accuracy of $0.2 \mathrm{~mm}$ was used. After recording the weight and height of the subjects, the body mass index was calculated through the following formula:

BMI $=$ weight $(\mathrm{kg}) /($ height $(\mathrm{m}) 2$

After giving all the necessary instructions about the basic principles in weight lifting by the bodybuilding coach, the subjects were introduced to the precautions, the determination of 1RM (one-repitition maximum) and the way of testing the salivary cortisol. These data collection and anthropometric measurements were done in the first day of exercise in the first week (Monday).With regards to the fact that they had enough experience about using weight, In the first week on Monday at $5 \mathrm{pm}$, subjects were asked to take part in the 1RM test to determine the maximum amount of weight. Later, on the following exercise days in the first week, second and third weeks they were assigned to have fitness exercises on Monday, Wednesday and Friday at $5 \mathrm{pm}$ and no exercise for the rest of the week. It should be mentioned that during the fitness exercise and the rest days, saliva samples of the subjects were obtained every 2 hours (from 6 am to $9 \mathrm{pm}$ ), in order to determine the level of Cortisol.

Measuring 1RM and evaluating their record, were submitted when they did it with their maximum effort and were not able to do one more. They started $1 \mathrm{RM}$ process with 12 repetitions and continued according 8, 6, 3and 1 repetition. If they were able to do one set of 1RM more, we allowed them to do to achieve a better record. The rest time between 12 and 8 repetition was 1 minute, but between 8 and 6 was 1.5 minute and between 6 and 3 was 2.5 minute and between 3 and 1 was around 4 minute (Willardson, 2008). 


\subsection{Sampling}

The saliva samples of the subjects were obtained during the rest day ( 24 hours of no exercise as the evidence) and the exercise day (as the experiment/test). In order to avoid the effect of the intervening factors, such as the effect of the food on the secretion of cortisol hormone, the saliva samples of the athletes were obtained every 2 hours, in 10 separate intervals from 6 am to $9 \mathrm{pm}$ during the day, while unstimulated. Saliva samples were obtained first at 6 am, and the subsequent samples were collected immediately after the fitness exercise, at 7, 7.30, 9 and 11 am and 1, 3, 5, 7 and 9 $\mathrm{pm}$. At each of these stages, the subjects were asked to first wash and clean their mouth and then to throw an amount of unstimulated saliva into the laboratory sampling tubes. The researcher monitored the process of sample collecting. However, the collected saliva samples were immediately transferred to the special/professional medical and pathology laboratory and were frozen at $-20 \mathrm{C}$. The Italian-made RADIM kit and the ELISA method were used to determine the level of the hormones.

\subsection{Exercise protocol}

The protocol of the fitness exercise is shown in the table below:

Table 1. Strength Training Program of fitness Exercises Athletes

\begin{tabular}{lll}
\hline Type of Training & Set & Rep. \\
\hline Warm up and Stretching & 4 & 15 \\
\hline Barbell Flat Bench Press & 4 & 15 \\
\hline Leg Press & 4 & 15 \\
\hline Barbell Biceps Curls & 4 & 15 \\
\hline Dumbbell squat & 4 & 15 \\
\hline Front Lat & 4 & 15 \\
\hline Cross Over & 4 & 15 \\
\hline Hamstring Machine & 4 & 15 \\
\hline Barbell Front Shoulder Press & 4 & 15 \\
\hline Crunch with Weight & 4 & 15 \\
\hline
\end{tabular}

A similar protocol has already been announced by Kraemer and his colleagues (Gettler et al., 2011). After 10-15 minutes of general warm up exercises and stretching, the subjects started the testing exercises which included four sets of 15 repetitions, with 1.5 minutes rest between each set. It should be mentioned that at this stage, the heartbeats of the subjects were calculated to be above 180 beats per minute. In order to determine the intensity of the exercise, the heartbeats of 7 of the randomly chosen subjects were calculated by the Heart Rate Calculator. In this exercise, both of the two types of fitness exercise instruments, the machines and the free weights were used. The cause and error method were used to perform the one-rep maximum test using modern body-building instruments and the below formula (Gettler et al., 2011):

$1 \mathrm{RM}=\mathrm{W}$ (lifted weights) / [1.0278 - ((repetition till tiredness) / 0.0278)]

\subsection{Data analysis}

Descriptive statistics including means and standard deviation were calculated to explore the data. Normality test (Kolmogorove - Smirnove ) and repeated measure ANOVA method were utilized, specifically in order to analyze the changes in the cortisol hormone during the day and comparing its level during the two resting and exercise days. All statistical analyses and the graphs were done through SPSS (17th version, SPSS Inc., Chicago, IL).

\section{Results}

The pre-test individual and physiological characteristics of the chosen fitness athletes are shown in table 2. In tables 3 and 4, first the statistical description of central factors and the distribution of variance of cortisol are respectively shown, while separating the resting and the exercise days; and then Repeated Measures ANOVA method and the following tests are used to analyze the research hypothesis and objectives.

According to the result of box $\mathrm{M}$ test the covariance matrix of the variant cortisol, in the control and the experimental groups were consistent ( $>>0.05$ ). Therefore, the Repeated Measure ANOVA were used for the inferential analysis of the data. The results of the Mauchly test showed that the condition of homogeneity of variance-covariance matrix for periodic daily salivary cortisol test was not effective $(p<0.05)$. Thus, the second column of Repeated Measure ANOVA and indeed the data from Greenhouse Gessier, will be used thereafter.

Table 3 shows the results of the analysis of the internal collective effects in the salivary cortisol variant.

The first row of table 3 shows that the comparison between the mean score of the control and the experimental groups, in pre and posttests, proved significant $(\mathrm{p}<0.001, \mathrm{~F}(9,252)=351.915)$.

In order to compare the rate and the amount of the variance between the groups, refer to the 2nd row of table 3 and the slope of their equivalent lines in Figure 1. The second row of the table 3 shows that the internal collective interaction (the slope of the lines) was significant $(\mathrm{F}(9,252)=4.856, \mathrm{p}<0.001)$. In other words, when the variance of the score of each group of the research is separately analyzed, the internal collective pattern of the variance can be seen to be meaningfully different. 
Table 1. Descriptive Statistic of Individual and Physiological Characteristics of the Participating Subjects (15 fitness athletes)

\begin{tabular}{lc}
\hline Characteristics & Mean \pm Standard deviation \\
\hline Age (years) & $20.35 \pm 1.20$ \\
\hline Weight $(\mathrm{kg})$ & $74.15 \pm 3.20$ \\
\hline Height $(\mathrm{cm})$ & $177.30 \pm 3.45$ \\
\hline RM $(\mathrm{kg} / \mathrm{mm})$ & $23.73 \pm 0.74$ \\
\hline Fat (percentage) & $14.48 \pm 2.05$ \\
\hline Fatty tissue $(\mathrm{kg})$ & $11.36 \pm 2.00$ \\
\hline Non-fatty tissue $(\mathrm{kg})$ & $11.36 \pm 2.00$ \\
\hline Resting heart rate (per minute) & $66.90 \pm 5.32$ \\
\hline Maximum heart rate (per minute) & $183.460 \pm 5.96$ \\
\hline Duration of exercise ( hours per week) & $8.58 \pm 2.25$ \\
\hline Professional /Experience in Body building (years) & $2.85 \pm 1.60$ \\
\hline
\end{tabular}

Table 3. Results of Repeated Measure Analysis of Variance for Sampling Time and Groups on Cortisol

\begin{tabular}{|c|c|c|c|c|}
\hline Source & $\begin{array}{l}\text { Degree of } \\
\text { freedom }\end{array}$ & Mean Square & F value & $\mathrm{P}$ value \\
\hline Sampling time & 4.264 & 5358.571 & 351.915 & $<0.001$ \\
\hline Group * Sampling time & 4.264 & 69.550 & 4.568 & $<0.001$ \\
\hline
\end{tabular}

Table 4. Between Subject (Groups) Effect

\begin{tabular}{cccccc}
\hline Source & Total Square & Freedom degree & Mean square & F & P value \\
\hline Group & 147.188 & 1 & 147.188 & 12.123 & 0.002 \\
\hline Error & 339.958 & 28 & 12.141 & & \\
\hline
\end{tabular}

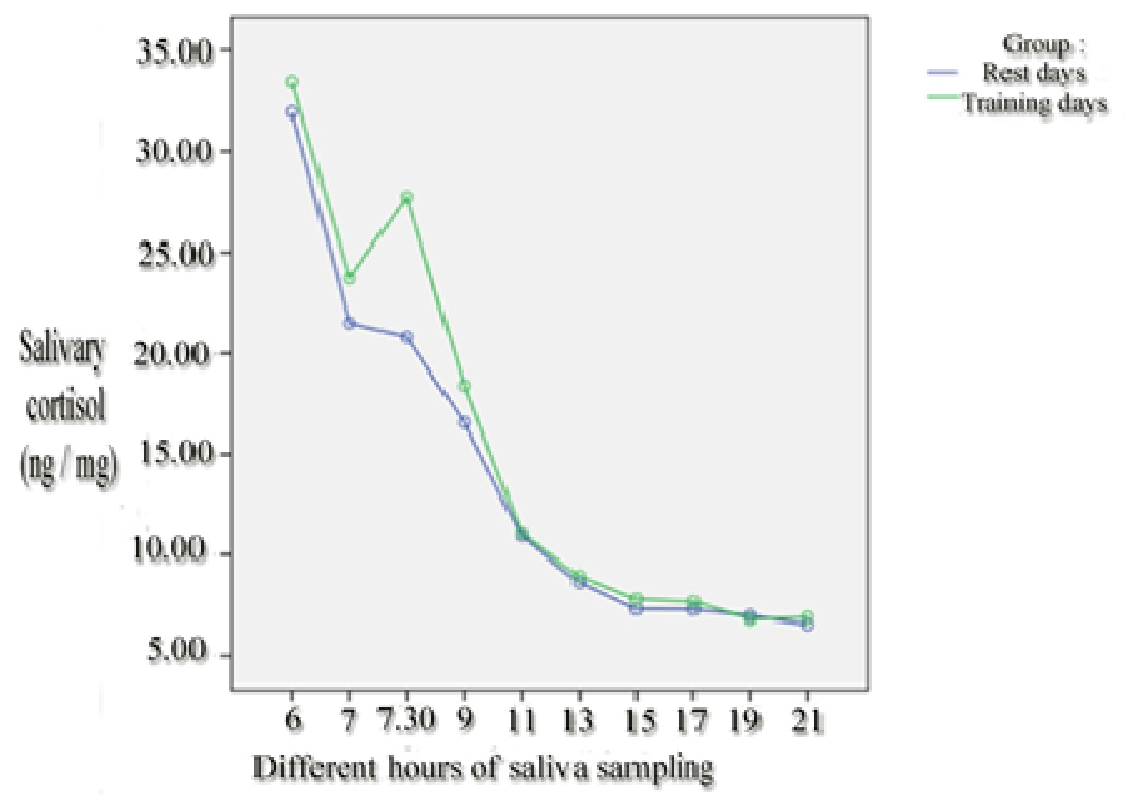

Figure1. Comparison between Changes in Circadian Rhythm of Salivary Cortisol in Rest and During Exercise at Different Times of Sampling

Comparison of salivary cortisol between experimental and control groups was done by using repeated measure Analysis of variance. They revealed that the amount of the salivary cortisol of both groups was significantly different $(\mathrm{F}=12.123$, $\mathrm{P}=0.002)$.

\section{Discussion}

The aim behind the current study is a comparison between salivary cortisol release in rest and training days in male fitness trainers. The findings show that the level of cortisol was at its highest in the beginning hours of the day (6 am) and reached to its lowest level in the following hours $(9 \mathrm{pm})$. This rhythm was consistent with the hypothesized rhythm for the secretion of cortisol. In the present study, independent sample $t$ test also showed that the overall rhythm/trend of the variance in the circadian salivary cortisol was the same in both the exercise and the resting days $(p>0.05)$. 
Although the results of the sampling in various times show that there was a significant increase in the level of the cortisol immediately after the exercise, but in the other hours of the day this variance was not significant, and followed similar rhythm in both the exercise and the resting days, as well as the other hours of the day (table 3).

The results of the present study also show that, in the exercise days with the fitness program, the fitness athletes had an increase in the level of the cortisol. This correspondence was at its strongest around $7.30 \mathrm{am}$, which seems to be the effect of the fitness program on the secretion of cortisol. Since this rhythm returned to its normal after the fitness exercise, and followed the same trend in both the exercise and the rest day on the other sampling times, and since there was no significant difference in all the other hours of the day $(\mathrm{p}>0.05)$, it can be said that although the fitness exercise has an effect on the synthesis and secretion of Cortisol ( Figure 1), it does not affect the total circadian rhythm. Results showed that a few hours after the omission of the fitness exercise, the rhythm returned to normal. The average of the level of cortisol was equal to the hypothesized circadian rhythm for the secretion of cortisol.

The secretion of cortisol is controlled by a stimulating hormone of the cortex of the adrenal gland, (Adrenocorticotropic hormone (ACTH)), which is itself produced in the anterior pituitary gland. This hormone, also called Adrenocorticotropy or Corticotropy, can also intensify the secretion of the adrenal Androgensby the adrenal cortex (Ghasemnezhad, 2011). The hypothalamus is mainly affected by many types of stress, which activates the system and results in a quick secretion of cortisol. Cortisol in turn, causes a series of metabolic changes in order to eliminate the harmful elements of the stressing conditions. Moreover, there is a direct feedback of cortisol to the hypothalamus and the anterior pituitary for maintaining the level of cortisol in the plasma, while the body is not under stressful condition. It should be noted that, stress is always a greater factor which can break down the direct controlling feedback of cortisol, and result in the secretion of cortisol at various times of the day (Ghasemnezhad, 2011).The stress caused by excitement or physical exercises, result in nervous responses from the outer sections of the body towards Hypothalamus. Then the hypothalamus secretes the Corticotropin-releasing factors that stimulate the anterior portion of pituitary for the secretion of ACTH. ACTH on the other hand, causes the secretion of cortisol from the adrenal cortex into blood (Daly and Rich, 1998).

The main effect of cortisol on metabolism is its capability of stimulating the gluconeogenes, which is the generation of carbohydrate from protein and other substances in the liver. The amount of gluconeogenes can increase up to 10 times during the day. Cortisol can also extract amino acids from the outer liver tissues, especially from muscles. As a result, there will be more amino acid in plasma to enter the gluconeogenesis process of the liver, and consequently, increases the secretion of glucose. Cortisol results in an average reduction in the consumption of the glucose by cells all over the body.

According to physiologists, cortisol directly delays the consumption of glucose, in a stage between its entrance and final stage of analysis. On the other hand, cortisol will result in the reduction of protein supplies in all body cells except the liver cells. The reason for this is the reduction in the synthesis and increase in the catabolism of the protein in the cells. Therefore, cortisol extracts the amino acids from the other tissues except the liver, and by doing this reduces the supply of the protein in the tissues. Cortisol raises the level of free fatty acids and their consumption in plasma, in order to enhance the generation of the energy. Cortisol also increases the oxidation of free fatty acids in cells as well, which can be the result of the reduction in the transition of glucose into the fatty cells (Ghasemnezhad, 2011).

Resistance exercises are recognized to be strong stimulators in the temporary increase of the masculine hormones. There are many other factors that can increase or decrease the level of hormones in the body (Shariat, Kargarfard Mehdi and Sharifi, 2011). Psychological pressures and physical exercises are among the strongest and the most influential stimulators. The results of the studies show that the quality of performing the exercises, in respect to the following variables, is critical in hormone responses: intensity and duration, type of the exercise (aerobic or anaerobic); fitness and gender of the subjects; rest intervals between the exercises, and the conditions under which the recovery of the muscles takes place. Thus, it can be said that each hormone follows a specific pattern in response to each type of the exercises (Webb, Rosalky, Tangsilsat, McLeod, Acevedo, 2013). Certain factors such as sleep or waking periods, nutrition, meal time, physical exercises, hormones and stress can influence and change the pattern of the circadian rhythm (Duclos et al., 2003). Fitness exercises without enough rest intervals and the stress caused by sports competitions in the long term, can result in changes in the physiological, immunological, psychological and functional status of the athletes (Fry et al., 2000).

In this situation, the level of the hormones undergoes some changes. For example, the level of the anabolic hormones such as testosterone decreases, while the level of catabolic hormones such as cortisol increases, and subsequently the body show a total tendency toward catabolism. (Gabriel, Urhausen, Valet, Heidelbach,1998). Michael and his colleagues purported that the intensity of the exercises has a primary and immediate influence on the secretion of cortisol up to $97 \%$ percent of its level in the rest days .It also had a significant difference with its level while light resistance exercises (Mcguigan et al., 2004). Immediately after the exercises, the level of these hormones increased in both men and women (Kraemer, Dziados, Marchitelli, Gordon, Harman, Mello, Fleck, Frykman PN, 1993) .

There is a significant change in the level of the saliva cortisol between the morning and evening, before the exercises, and the salivary cortisol in the rest $(\mathrm{p}<0.05)$. Physical exercise can significantly increase the level of cortisol, and as the final product, the hypothalamus-hypophysis-adrenal axis can be stimulated during and by the intensity of the exercise (Dimitriou and Sharp , 2002). Apart from that doing the resistance exercises in the evening, in comparison with morning exercise, can positively affect the cortisol response and the testosterone-cortisol ratio T/C. The level of cortisol had significantly reduced before the exercise in the evening $(\mathrm{p} \leq 0.05)$, and resulted in the increase in the testosterone- 
cortisol ratio. This shows that physical exercises affect the hormone response, and by considering the circadian rhythm we can achieve the ideal metabolism and improve the consistency between skeletal muscles and physical resistance exercises (Men, 2004). In endurance training, there is much less change in the level of testosterone and cortisol, in comparison with those who had resistance exercises and testosterone and cortisol responses are more associated with the manner, the type and intensity of the exercises (Paffenbarger, Blair, \& Lee, 2001).

According to the aforementioned information's, it seems that the results of the present study, with regard to cortisol, are in agreement with the findings of most of the previous researches.

It seems that the main reason for the increase in the level of cortisol, under stressful condition and physical pressure, is the change in the function of the hypothalamus- hypophysis- adrenal axis (Banfi, 2006). The hypothalamushypophysis- adrenal axis is stimulated in response to fitness exercises and stressful situations and increases its function, this results in an increase in the secretion of ACTH and subsequently an increase in the secretion of cortisol (Figure 2).

\section{Conclusion}

Regarding the advantages of blood cortisol analysis over the analysis of saliva cortisol, as well as the high consistency found between saliva and plasma, in the level of cortisol and testosterone ( $r>0.9)$ (Rosmond et al., 2003) as the result of the previous studies, the evaluation and measurement of these hormones, especially saliva cortisol, can be regarded as a well-selected, helpful method in primary researches and clinical environments. It can also serve as a good measure in programming the sessions for fitness exercises and for bodybuilding. The results of the study show that fitness exercises have a significant effect on the synthesis and secretion of saliva cortisol, but they have no effect on the circadian rhythm of saliva cortisol in the waking status of male fitness athletes.

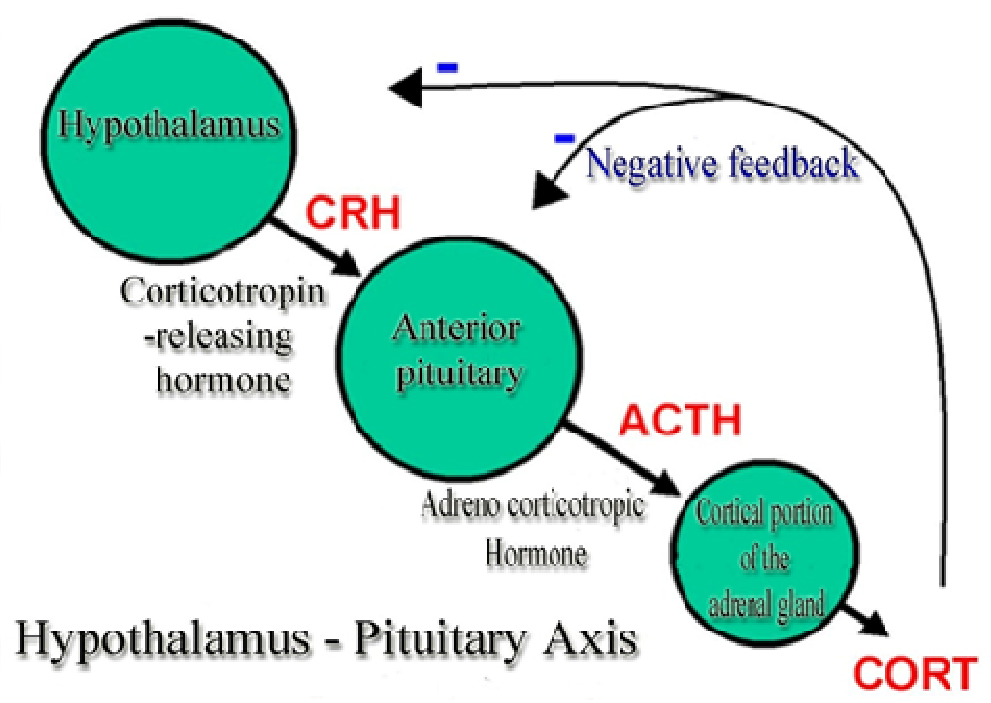

Figure 2. Hypothalamus- Pituitary- Adrenal Axis and its Mechanism of Action

\section{Acknowledgement}

Special thanks to Prof. David Pyne for his really helpful comments, to Dr. Mahmoud Danaee for his guidance through the statistical analysis of this study, and our editorial board Rachel Hua Yeh Fung and Roya Jabarouti.

\section{References}

Acevedo EO, Dzewaltowski D, Kilgore JL, Kraemer GR, C. VD. (1996). Effects of low-volume resistive exercise on. Int J Sports MED.

Banfi G, D. A. (2006). Free testosterone/cortisol ratio in soccer: usefulness of a categorization of values. J Sports MED Phys Fitness.

C Bosco, R Colli, R Bonomi, S P Von Duvillard, A. V. (2000). Monitoring strength training now. Medicine \& Science in Sports \& Exercise.

Daly RM, Rich PA, K. R. (1998). Hormonal responses to physi. Eur J Appl Physiol Occup Physiol.

Dimitriou L, Sharp NC, D. M. (2002). Circadian effects on the acute responses of salivary cortisol and IgA in well trained swimmers. Br J Sports MED.

Duclos, M., Gouarne, C., \& Bonnemaison, D. (2003). Acute and chronic effects of exercise on tissue sensitivity to glucocorticoids. Journal of applied physiology (Bethesda, Md. : 1985), 94 (3), 869-75.

Fry, A. C., Kraemer, W. J., Stone, M. H., Perry Koziris, L., Thrush, J. T., \& Fleck, S. J. (2000). Relationships Between Serum Testosterone, Cortisol, and Weightlifting Performance. The Journal of Strength and Conditioning Research, 14 (3), 338 . 
Gabriel HH, Urhausen A, Valet G, Heidelbach U, K. W. (1998). Overtraining and immune system a prosp. MED Sci Sports Exerc.

Gettler, L. T., McDade, T. W., \& Kuzawa, C. W. (2011). Cortisol and testosterone in Filipino young adult men: evidence for co-regulation of both hormones by fatherhood and relationship status. American journal of human biology: the official journal of the Human Biology Council, 23 (5), 609-20. doi:10.1002/ajhb.21187.

Ghasemnezhad, M. (2011). Overtraining in Sport: Physiological, Psychological and Performance Effects of Participation in Division I Competitive Basketball, 05 (04), 195-206.

Kraemer WJ, Dziados JE, Marchitelli LJ, Gordon SE, Harman EA, Mello R, Fleck SJ, Frykman PN, T. N. (1993). Effects of different heavy-resistance exercise protocols on plasma beta-endorphin concentrations. J Appl Physiol.

Mcguigan, M. R., Egan, A. D., \& Foster, C. (2004). Salivary cortisol responses and perceived exertion during high intensity and low intensity bouts of resistance exercise, (2003), 8-15.

Men, I. of circadian time structure on acute hormonal responses to a single bout of heavy-resistance exercise in weighttrained. (2004). Influence of circadian time structure on acute hormonal responses to a single bout of heavy-resistance exercise in weight-trained men.

Paffenbarger, R. S., Blair, S. N., \& Lee, I. M. (2001). A history of physical activity, cardiovascular health and longevity: the scientific contributions of Jeremy N Morris, DSc, DPH, FRCP. International journal of epidemiology, 30 (5), 118492. Retrieved from http://www.ncbi.nlm.nih.gov/pubmed/11689543

Rosmond, R., Wallerius, S., Wanger, P., Martin, L., Holm, G., \& Björntorp, P. (2003). A 5-year follow-up study of disease incidence in men with an abnormal hormone pattern. Journal of internal medicine, 254(4), 386-390.

Sciences, H. (2011). Circadian rhythms in exercise performance : Implications for hormonal and muscular adaptation, (December), 600-606.

Shariat Ardalan, K. M., \& Sharifi, G. R. The Effect of heavy resistance exercise on circadian rhythm of salivary cortisol in male body building atheletes. journal of isfahan medical school (I.U.M.S), 29(167), 0.

Webb HE, Rosalky DS, Tangsilsat SE, McLeod KA, Acevedo EO, W. B. (2013). Aerobic fitness affects cortisol responses to concurrent challenges. MED Sci Sports Exerc.

Willardson, J. M. (2008). A Brief Review: How Much Rest between Sets?.Strength \& Conditioning Journal, 30(3), 4450. 